\title{
Effect of field tilting on the vortices in irradiated Bi-2212.
}

\author{
V. Ta Phuoc, A. Ruyter, R. de Sousa, L. Ammor, E. Olive, J.C. Soret ${ }^{\text {a }}$ \\ a Laboratoire d'Electrodynamique des Matériaux Avancés. \\ Université F.Rabelais - UFR Sciences - Parc de Grandmont. \\ 37200 Tours- France.
}

We report on transport measurements in a Bi-2212 single crystal with columnar defects parallel to the c-axis. The tilt of the magnetic field away from the direction of the tracks is studied for filling factors $f=B_{z} / B_{\phi}<1$. Near the Bose Glass transition temperature $T_{B G}$, the angular scaling laws are verified and we find the field independent critical exponents $\nu^{\prime}=1.1$ and $z^{\prime}=5.30$. Finally, above $H_{\perp C}$ we evidence the signature of a smetic-A like vortex phase. These experimental results provide support for the Bose Glass theory.

Columnar defects have been introduced in HTCS in order to avoid dissipation due to vortex motion. In the case of parallel tracks Nelson and Vinokur 1] have predicted a transition between a so-called Bose Glass (BG) and vortex liquid, at a critical temperature $T=T_{B G}$. An important result is that field tilting does not destabilize the BG phase. Above a threshold transverse field $H_{\perp C}(T)$ perpendicular to the columnar defects $(\mathrm{CD})$, the flux lines accomodate simultaneously to CD and to the transverse field direction, defining a smectic-A like phase as proposed by Hwa and Nelson[2] Increasing further $H_{\perp}$ leads to a vortex-liquid state [3]. Recently, Grigera et al. [4] have evidenced a threshold transverse field $H_{\perp C}(T)$ with resistivity measurements performed on a twinned Y-123 single crystal.

In this paper, we present results obtained on a Bi-2212 single crystal, irradiated with CD parallel to the c-axis with $5.8 \mathrm{GeV} \mathrm{Pb}$ ions in GANIL (Caen- France). The CD density corresponds to a matching field $B_{\phi}=0.75 \mathrm{~T}$. Isothermal $\mathrm{I}-\mathrm{V}$ curves have been obtained varying the tilt angle between the magnetic field and the c-axis of the crystal. Measurements were performed keeping the $B_{z}$ component constant, where the z-axis is along the c-axis of the sample. In the insert of Fig. 1, the log-log plot of isothermal ohmic resistance versus $H_{\perp} / H_{z}$ is displayed above and below $T_{B G}$. For $T>T_{B G}$, ohmic behaviour is detected, within the experimental sensitivity, even for vanishing transverse field. In contrast, for $T<T_{B G}$ the ohmic resistance goes to zero at some critical tilt showing the existence of a critical transverse magnetic field $H_{\perp C}(T)$. In the BG theory, scaling functions $f_{+}$and $f_{-}$are predicted to describe the resistivity in the presence of a tilted magnetic field, above and below $T_{B G}$, respectively. The resistance then reads:

$R \sim|t|^{\nu^{\prime}\left(z^{\prime}-2\right)} f_{ \pm}\left(\left(H_{\perp} / H_{z}\right)|t|^{-3 \nu^{\prime}}\right)$.

where $t=\left(T-T_{B G}\right) / T_{B G}$ is the reduced temperature, and $\nu^{\prime}$ and $z^{\prime}$ are critical exponents. Fig. 1 displays such scaling properties. We find that the scaling functions $f_{+}$and $f_{-}$collapse for all the filling fractions $f=B_{z} / B_{\phi}<1$ investigated in our experiment. Moreover, we obtain field independent critical exponents, $\nu^{\prime}=1.1 \pm 0.1$ and $z^{\prime}=5.30 \pm 0.05$. These results are in good agreement with the BG theory [1] and other experimental results 四 6 .

We shall now consider the case $T<T_{B G}$. For $H_{\perp}>H_{\perp C}(T)$, the vortex motion is mediated by kinks aligned in chains in the direction of the transverse magnetic field $H_{\perp}$ [2], in such a way that the chain density is directly related to the linear resistance:

$R \propto n_{\text {chain }} \sim\left(H_{\perp}-H_{\perp C}(T)\right)^{3 / 2}$.

Scaling arguments lead to a critical transverse magnetic field $H_{\perp C}(T)$ vanishing as $T \rightarrow T_{B G}^{-}$ as:

$H_{\perp C}(T) \sim|t|^{3 \nu^{\prime}}$. 


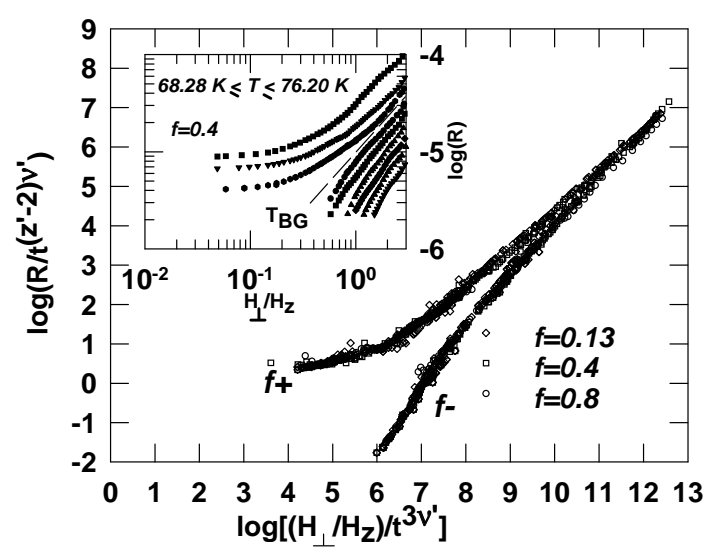

Figure 1. Scaling properties according to Eq. 1 for different filling fractions $f=0.13, f=0.4$, and $f=0.8$. The field independent critical exponents we obtain are $\nu^{\prime}=1.1 \pm 0.1$ and $z^{\prime}=$ $5.30 \pm 0.05$. The insert displays a log-log plot of isothermal ohmic resistance versus $H_{\perp} / H_{z}$ above and below $T_{R \Omega}$ for $f=0.4$.

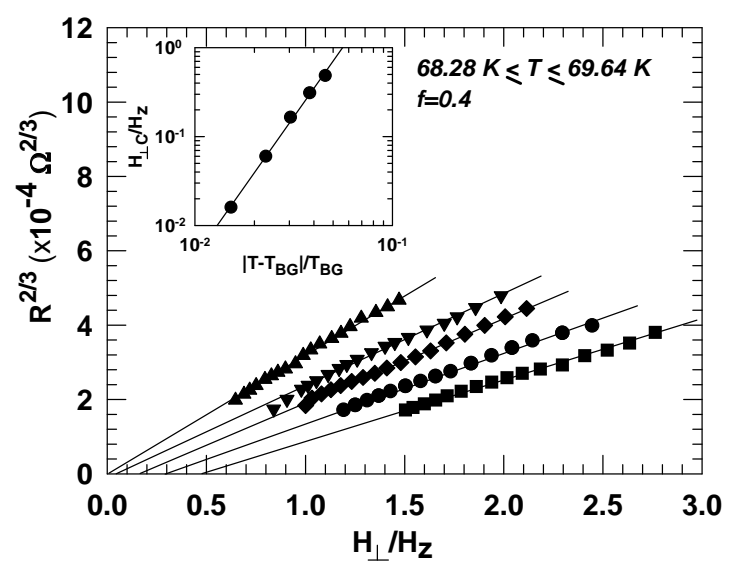

Figure 2. Plot of $R^{2 / 3}$ versus $H_{\perp} / H_{z}$. The data exhibit linear behaviour as expected in Eq. 2. The intersections of the lines with the abscissa axis give the critical transverse fields for different temperatures. The insert displays the transverse critical field $H_{\perp c} / H_{z}$ versus the reduced temperature. The slope gives directly the critical exponent value $\nu^{\prime}=1.1 \pm 0.1$.
Figure 2 displays a plot of $R^{\frac{2}{3}}$ versus $H_{\perp} / H_{z}$. The solid lines are successfull fits of Eq. 2 to data. This result does support the existence of a smectic-A behaviour. The intersection of a linear fit with the abscissa-axis directly gives the critical transverse magnetic field $H_{\perp C}(T)$ at a given temperature. The insert of Fig. 2 shows a log-log plot of $H_{\perp C}(T)$ thus obtained versus the reduced temperature $t=\left(T-T_{B G}\right) / T_{B G}$. The solid line represents a least-square fit of Eq. 3 to data. According to Eq. 3, we find therefrom the critical exponent value $\nu^{\prime}=1.1 \pm 0.1$. Note that this value is consistent with the one we found above by another way, as predicted by Nelson and Vinokur 迎.

In conclusion, we have investigated the Bose Glass phase transition on an irradiated Bi-2212 single crystal versus both the filling fraction $f=$ $B_{z} / B_{\phi}<1$ and the magnetic field tilt. We have shown that the Bose Glass transition in the presence of a tilted field verifies the scaling rules predicted by Nelson and Vinokur with both field independent scaling functions and critical exponents $\nu^{\prime}=1.1$ and $z^{\prime}=5.30$. A smectic-A like behaviour has been evidenced. Finally, the critical tilt $H_{\perp C} / H_{z}$, separating this phase from the Bose Glass one, has been found to vary as $H_{\perp C} / H_{z} \sim|t|^{3 \nu^{\prime}}$ in agreement with the theorical expectation of a sharp cusp in the $T-H_{\perp} / H_{z}$ phase diagram.

\section{REFERENCES}

1. D. R. Nelson, V. M. Vinokur, Phys. Rev. B 48 (1993), 13060.

2. T. Hwa, D.R. Nelson, Phys. Rev. B 48 (1993), 1167.

3. N. Hatano, D.R. Nelson, Phys. Rev. Lett. 77 (1996), 570.

4. S.A. Grigera, E. Morré, E. Osquiguil, C. Balseiro, G. Nieva, F. de la Cruz, Phys. Rev. Lett. 81 (1998), 2348.

5. J.C. Soret, V. Ta Phuoc, L. Ammor, A. Ruyter, R. De Sousa, E. Olive, G. Villard, A. Wahl, Ch. Simon, accepted in Phys. Rev. B.

6. V. Ta Phuoc, A. Ruyter, L. Ammor, A. Wahl, J.C. Soret, Phys. Rev. B 56 (1997), 122. 